\title{
CHARACTERS OF AUTOMORPHISM GROUPS ASSOCIATED WITH KÄHLER CLASSES AND FUNCTIONALS WITH COCYCLE CONDITIONS
}

\author{
Akito Futaki and Yasuhiro Nakagawa
}

\section{Introduction}

Let $M$ be a connected compact Kähler manifold. An obvious necessary condition for $M$ to admit a Kähler-Einstein metric is that the first Chern class $c_{1}(M)$ is either negative, zero or positive, where a real 2-dimensional de Rham cohomology class is said to be negative (resp. positive) if it is represented by a negative (resp. positive) definite $(1,1)$-form. Conversely, if $c_{1}(M)$ is negative or zero then $M$ admits a Kähler-Einstein metric by the solution to the Calabi conjectures (Aubin [1], Yau [21]).

In the remaining case where $c_{1}(M)$ is positive, in which case $M$ is often called a Fano manifold, there are further necessary conditions. First of all the Lie algebra $\mathfrak{h}(M)$ of all holomorphic vector fields on a Kähler-Einstein Fano manifold $M$ is reductive (Matsushima [14]). Secondly a Lie algebra character $f: \mathfrak{h}(M) \rightarrow C$ introduced in [10] must vanish on a Kähler-Einstein Fano manifold. It was also proven by Bando-Mabuchi [5] that if $M$ admits a KählerEinstein metric then certain functional, called K-energy, of $M$ is bounded from below. This analytic necessary condition played a theoretically important role in the later studies. In fact Ding and Tian [9] extended the results of [10] and [5] to obtain a necessary condition applicable to manifolds which do not carry any non-zero holomorphic vector fields. Tian [20] further extended these ideas to define certain notions of stability, called K-stability and CM-stability, and presented an example of a Fano manifold with no non-zero holomorphic vector fields and no Kähler-Einstein metrics. On the other hand there are known sufficient conditions for the existence of positive Kähler-Einstein metrics by Aubin [2], Ding [8], Siu [17], Tian [18] and Nadel [15].

Now one would hope to have a necessary and sufficient condition for the existence of positive Kähler-Einstein metrics. To state such a condition, Tian [20] introduced a notion of properness for the K-energy and a functional introduced by Ding [8]. Combining [20] and [4] one can show, at least when $\mathfrak{h}(M)=0$, that a Fano manifold admits a Kähler-Einstein metric if and only if

Received November 24, 1999; revised July 18, 2000. 
Ding's functional (or K-energy) is proper, and thus the properness of Ding's functional and the properness of K-energy are equivalent. It can be checked directly that if the sufficient condition obtained in, for example, [18] is satisfied then the both functionals are proper, and that if either of the functionals is proper then the necessary conditions obtained in [10], [9], [20] are satisfied.

The purpose of this paper is to show that there is a family of functionals satisfying cocyle conditions and that both of the K-energy and Ding's functional can be derived from the family. It is constructed as follows. Given a Kähler manifold $M$ with a Kähler class $\Omega$, there exists a Lie algebra character $f_{\Omega}: \mathfrak{h}(M) \rightarrow C$ with the property that if $M$ admits a Kähler metric of constant scalar curvature in $\Omega$ then $f_{\Omega}=0$ (cf. [11], [7], [3]). Of course when $M$ is a Fano manifold and $\Omega=c_{1}(M)$, then $f_{\Omega}$ coincides with the above $f$. We try to lift $f_{\Omega}$ to a character of the group of automorphisms which preserve $\Omega$. This can be performed successfully when $\Omega$ is a Hodge class and the group action lifts to a holomorphic line bundle $L$ whose Chern class coincides with $\Omega$, see Nakagawa [16]. We can give an explicit formula of the character in terms of the ChernSimons invariants of certain virtual bundles. A merit of this formula is that it is written using Hermitian metrics of $L$ and the anti-canonical bundle $K_{M}^{-1}$ and that we may choose these two metrics independently. Note that if in certain situations the character can be written in terms of a Kähler form $\omega$ and the pull-back form $\omega^{\prime}=\sigma^{*} \omega$ by an automorphism $\sigma$, then we obtain a functional written in terms of $\omega$ and $\omega^{\prime}$ satisfying cocycle conditions.

Now return to the situation where $\Omega=c_{1}(M)$ and $L=K_{M}^{-1}$. In section 4 we will see that if we choose a Kähler form $\omega \in \Omega$ and then choose a fiber metric of $L$ so that its Chern form is equal to $\omega$, then the formula of the group character yields the K-energy. On the other hand if we choose metrics for $L$ and $K_{M}^{-1}$ equal, the formula yields Ding's functional.

\section{Review of characteristic classes of foliations}

A transeversely holomorphic foliation $\mathscr{F}$ of complex codimension $m$ on a smooth manifold $W$ of real dimension $2 m+n$ is given by a system of local charts $\left\{z^{1}, \ldots, z^{m}, x^{1}, \ldots, x^{n}\right\}$ where $\left\{x^{1}, \ldots, x^{n}\right\}$ is real coordinates along the leaves and $\left\{z^{1}, \ldots, z^{m}\right\}$ are complex coordinates in the normal directions, such that for any neighboring local charts $\left\{w^{1}, \ldots, w^{m}, y^{1}, \ldots, y^{n}\right\}$, the $w^{i}$ s are holomorphic functions of $z^{i}$ s. Then there is a subbundle $T_{1,0}^{*}$ of $T W^{*} \otimes C$ spanned by $\left\{d z^{1}, \ldots, d z^{m}\right\}$ in local charts. Note that the definition of $T_{1,0}^{*}$ is independent of the choice of local charts. A section of $T_{1.0}^{*}$ will be called a differential form of type $(1,0)$. Let $S$ be the subbundle of $T W \otimes C$ annihilated by $T_{1,0}^{*}$. The quotient bundle $v(\mathscr{F})=(T W \otimes C) / S$ is called the normal bundle of $\mathscr{F}$.

Let $E \rightarrow W$ be a complex vector bundle of rank $r$ over $W$. A basic connection of $E$ is a linear connection whose connection form is of type $(1,0)$. Not every vector bundle admits a basic connection. But, for example, an argument using partition of unity shows that the normal bundle $v(\mathscr{F})$ carries basic 
connections ([6]). It is obvious from the dimension reasons that, for a multiindex $\alpha$ with $|\alpha|>m$, the Chern form $c^{\alpha}(E, \nabla)$ vanishes identically if $\nabla$ is a basic connection. Thus we can define characteristic classes of foliations, which we review next.

The differential graded algebra $W U_{m}$ is defined as

$$
W U_{m}=\bigwedge\left(u_{1}, \ldots, u_{m}\right) \otimes\left\{\boldsymbol{C}\left[c_{1}, \ldots, c_{m}\right] / \operatorname{deg}>2 m\right\} \otimes\left\{\boldsymbol{C}\left[\bar{c}_{1}, \ldots, \bar{c}_{m}\right] / \operatorname{deg}>2 m\right\}
$$

where $\bigwedge\left(u_{1}, \ldots, u_{m}\right)$ is the exterior algebra in $u_{1}, \ldots, u_{m}$ with $\operatorname{deg} u_{i}=i$, $\boldsymbol{C}\left[c_{1}, \ldots, c_{m}\right]$ is a polynomial algebra in $c_{1}, \ldots, c_{m}$ with $\operatorname{deg} c_{i}=2 i$ and similarly for $\boldsymbol{C}\left[\bar{c}_{1}, \ldots, \bar{c}_{m}\right]$, and where the differential $d$ is defined by $d u_{i}=c_{i}-\bar{c}_{i}$ and $d c_{i}=d \bar{c}_{i}=0$.

Suppose that $E$ carries basic connections. Let $\Omega^{*} W$ be the de Rham complex of $W$. We define a differential graded algebra map $\lambda_{W}: W U_{m} \rightarrow \Omega^{*} W$ as follows.

Choose an Hermitian metric of $E$ and take an arbitrary metric connection $\nabla^{0}$, and a basic connection $\nabla^{1}$ of $E$. Let $p: W \times I \rightarrow W$ be the projection, where $I$ denotes the unit interval. Then $\nabla^{0,1}=s \nabla^{1}+(1-s) \nabla^{0}$ is a connection of $p^{*} E$. Denote by $c_{i}\left(\nabla^{0}\right), c_{i}\left(\nabla^{1}\right)$ and $c_{i}\left(\nabla^{0,1}\right)$ respectively the $i$-th Chern forms with respect to $\nabla^{0}, \nabla^{1}$ and $\nabla^{0,1}$. Set $h_{i}=p_{*} c_{i}\left(\nabla^{0,1}\right)$. Then we have

$$
d h_{i}=c_{i}\left(\nabla^{1}\right)-c_{i}\left(\nabla^{0}\right),
$$

and

$$
d h_{i}-d \bar{h}_{i}=c_{i}\left(\nabla^{1}\right)-\overline{c_{i}\left(\nabla^{1}\right)}
$$

since $\nabla^{0}$ is a metric connection and its Chern forms are real forms. From this it follows that the map $\lambda_{W}$ defined by

$$
\lambda_{W}\left(u_{i}\right)=h_{i}-\bar{h}_{i}, \quad \lambda_{W}\left(c_{i}\right)=c_{i}\left(\nabla^{1}\right), \quad \lambda_{W}\left(\bar{c}_{i}\right)=\overline{c_{i}\left(\nabla^{1}\right)}
$$

is a DGA-map. It is well-known that the induced homomorphism $\lambda_{W}^{*}$ : $H^{*}\left(W U_{m}\right) \rightarrow H_{\mathrm{D} R}^{*}(W ; \boldsymbol{C})$ is independent of the choice of the Hermitian connection $\nabla^{0}$ and the basic connection $\nabla^{1}$. We note that $\sum_{k=0}^{m} c_{1}^{k} u_{1} \bar{c}_{1}^{m-k}$ is closed in $W U_{m}$, and thus we have

$$
\lambda_{W}^{*}\left(\sum_{k=0}^{m} c_{1}^{k} u_{1} \bar{c}_{1}^{m-k}\right) \in H^{2 m+1}(W ; \boldsymbol{C}) .
$$

\section{The case of suspension foliations}

Let $M$ be a compact Kähler manifold and $\sigma$ an automophism of $M$. Suppose that $\sigma$ generates an infinite cyclic group $G \cong \boldsymbol{Z}$. Let $E \rightarrow M$ be a holomorphic vector bundle. We assume that the action of $G$ lifts to $E$. We set $E_{\sigma}:=(\boldsymbol{R} \times E) / G$ and $M_{\sigma}:=(\boldsymbol{R} \times M) / G$, where $G$ acts on $\boldsymbol{R} \times E$ by

$$
\sigma^{n}(v, t)=\left(t-n, \sigma^{n}(v)\right)
$$


and on $\boldsymbol{R} \times M$ similarly. There is a natural transversely holomorphic foliation on $\boldsymbol{R} \times M$ with leaf dimension 1 , and it descends to $M_{\sigma}$.

Lemma 3.1. The complex vector bundle $E_{\sigma} \rightarrow M_{\sigma}$ carries basic connections.

Proof. $E_{\sigma}$ is obtained as follows. Consider $I \times E$ and identify $\{1\} \times E$ and $\{0\} \times E$ by the relation $(1, v) \sim(o, \sigma(v)) . \quad M_{\sigma}$ is also obtained similarly. The leaves of the foliation are of the form $\{(t, p) \mid t \in I\}$. Remark that the vector bundle $E_{\sigma}$ is flat along the leaf direction, i.e. the transition functions do not involve the leaf coordinate.

Let $\phi(t)$ be a smooth function on $I$ such that $\phi(t) \equiv 0$ near $t=0$ and $\phi(t) \equiv 1$ near $t=1$. Choose any Hermitian metric $h$ of the line bundle $E \rightarrow M$. We define an Hermitian metric of $E_{\sigma} \rightarrow M_{\sigma}$ by

$$
\tilde{h}_{t}=(1-\phi(t)) h+\phi(t) \sigma^{*} h .
$$

Then by the above remark $\tilde{h}_{t}^{-1} \partial \tilde{h}_{t}$ defines a basic connection, where $\partial$ denotes the $(1,0)$-part of the exterior differentiation, namely

$$
\partial=\sum_{i=1}^{m} d z^{i} \frac{\partial}{\partial z^{i}}
$$

it terms of normal holomorphic coordinates $z^{1}, \ldots, z^{m}$.

So we can define $\hat{f}_{E}: G \rightarrow \boldsymbol{R}$ by

$$
\hat{f}_{E}(\sigma)=i \lambda_{W}^{*}\left(\sum_{k=0}^{m} c_{1}^{k} u_{1} \bar{c}_{1}^{m-k}\right)[W] .
$$

Recall that, given an Hermitian metric $h$ on a holomorphic vector bundle $E$, the Ricci form $\rho_{h}$ is given by

$$
\rho_{h}:=\frac{i}{2 \pi} \bar{\partial} \partial \log \operatorname{det} h .
$$

It represents the first Chern class $c_{1}(E)$, and its coefficients

$$
R_{i \bar{j}}:=-\frac{\partial^{2}}{\partial z^{i} \partial \bar{z}^{j}} \log \operatorname{det} h
$$

are called the Ricci curvature of $h$. When an automorphism $\sigma$ of $M$ lifts to an action on $E$, we have

$$
\sigma^{*} \rho_{h}=\rho_{\sigma^{*} h} .
$$

THEOREM 3.2. Let $h$ be an Hermitian metric of the holomorphic vector bundle $E \rightarrow M$. Then $\hat{f}_{E}(\sigma)$ can be given by 


$$
\hat{f}_{E}(\sigma)=\frac{1}{2 \pi} \int_{M} \log \frac{\operatorname{det} \sigma^{*} h}{\operatorname{det} h} \sum_{k=0}^{m} \sigma^{*} \rho_{h}^{k} \wedge \rho_{h}^{m-k} .
$$

Moreover $\hat{f}_{E}: G \rightarrow \boldsymbol{R}$ is a group character.

Remark 3.3. From the independence of the choice of Hermitian connections $\nabla^{0}$ and basic connections $\nabla^{1}$, the above expression of $f_{E}$ is also independent of the choice of Hermitian metrics $h$.

Proof. Let $\tilde{h}_{t}$ be as in the proof of Lemma 3.1, and put

$$
h_{t}=\left(\frac{(\operatorname{det} h)^{1-\phi(t)}\left(\operatorname{det} \sigma^{*} h\right)^{\phi(t)}}{\operatorname{det} \tilde{h}_{t}}\right)^{1 / r} \tilde{h}_{t},
$$

where $r$ is the rank of $E$. Then the Ricci form $\rho_{h_{t}}$ is written as

$$
\rho_{h_{t}}=(1-\phi(t)) \rho_{h}+\phi(t) \rho_{\sigma^{*} h} .
$$

It is obvious that

$$
\theta^{1}=h_{t}^{-1} \partial h_{t}
$$

also defines a basic connection, and it is easy to check that

$$
\theta^{0}=h_{t}^{-1} \partial h_{t}+\frac{1}{2} h_{t}^{-1} \partial_{t} h_{t}
$$

defines an Hermitian connection, where

$$
\partial_{t}=d t \frac{\partial}{\partial t}
$$

From the definition of $u_{1}$ we easily get

$$
\lambda_{W}^{*} u_{1}=\frac{i}{2 \pi} \partial_{t} \log \operatorname{det} h_{t}
$$

We also have

$$
\lambda_{W}^{*} c_{1}=\frac{i}{2 \pi}\left(\partial_{t}+\bar{\partial}\right) \partial \log \operatorname{det} h_{t}
$$

It follows that, for all $k$, we have

$$
\lambda_{W}^{*}\left(c_{1}^{k} u_{1} \bar{c}_{1}^{m-k}\right)=\frac{i}{2 \pi} \partial_{t} \log \operatorname{det} h_{t} \wedge\left(\frac{i}{2 \pi} \bar{\partial} \partial \log \operatorname{det} h_{t}\right)^{m} .
$$

From (3) and (4) we have 


$$
\begin{aligned}
i \lambda_{W}^{*} c_{1}^{k} u_{1} \bar{c}_{1}^{m-k}[W] & =(m+1) \frac{1}{2 \pi} \int_{M \times I} \phi^{\prime}(t) \log \frac{\operatorname{det} \sigma^{*} h}{\operatorname{det} h} d t \wedge\left(\rho_{h}+\phi(t)\left(\rho_{\sigma^{*} h}-\rho_{h}\right)\right)^{m} \\
& =(m+1) \frac{1}{2 \pi} \int_{M} \log \frac{\operatorname{det} \sigma^{*} h}{\operatorname{det} h} \sum_{k=0}^{m}\left(\begin{array}{c}
m \\
k
\end{array}\right) \frac{1}{k+1}\left(\rho_{h}\right)^{m-k} \wedge\left(\rho_{\sigma^{*} h}-\rho_{h}\right)^{k} \\
& =\frac{1}{2 \pi} \int_{M} \log \frac{\operatorname{det} \sigma^{*} h}{\operatorname{det} h} \sum_{k=0}^{m} \rho_{h}^{k} \wedge \rho_{\sigma^{*} h}^{m-k} .
\end{aligned}
$$

This completes the proof of the first half.

From (2) and the fact that the right hand side of (2) is independent of the choice of the metric $h$ it is easy to check

$$
\hat{f}_{E}(\sigma \tau)=\hat{f}_{E}(\sigma)+\hat{f}_{E}(\tau) .
$$

This completes the proof of the second half. An alternate proof can also be given as follows. There is a fibration $p: W=M_{G}=E G \times_{G} M \rightarrow B G=S^{1}$. The integration over the fibers gives

$$
p_{*}\left(i \lambda_{W}^{*} c_{1}^{k} u_{1} \bar{c}_{1}^{m-k}\right) \in H^{1}(B G ; \boldsymbol{R}) \cong \operatorname{Hom}(G, \boldsymbol{R}) .
$$

The interpretation of the last isomorphism shows that $\hat{f}_{E}$ is a homomorphism.

\section{A Lie algebra character and its lifting to a group character}

In this section we first review the Lie algebra character obtained as an obstruction to the existence of Kähler metric of constant scalar curvature ([11], [7], [3]), and give an explicit formula of its lifting to a group character.

Let $\Omega$ be a fixed Kähler class on an $m$-dimensional connected compact Kähler manifold $M$, and $\omega=i /(2 \pi) \sum g_{i j} d z^{i} \wedge d \bar{z}^{j}$ a Kähler form which represents $\Omega$. The Ricci form of $\omega$ will be denoted by

$$
\rho_{\omega}=\frac{i}{2 \pi} \sum R_{i \bar{j}} d z^{i} \wedge d \bar{z}^{j}=\frac{i}{2 \pi} \bar{\partial} \partial \log \operatorname{det} g .
$$

We put

$$
\begin{gathered}
V:=\Omega^{m}[M], \\
\mu=\frac{1}{V} \int_{M} \frac{s_{\omega}}{m} \omega^{m},
\end{gathered}
$$

where $s_{\omega}=g^{i \bar{j}} R_{i \bar{j}}$ denotes the scalar curvature of $\omega$. Then there is a smooth function $F_{\omega}$ uniquely determined up to constant such that

$$
s_{\omega}-\mu m=\Delta F_{\omega} .
$$

Define $f: \mathfrak{h}(M) \rightarrow \boldsymbol{C}$ by 


$$
f(X)=\frac{1}{2 \pi} \int_{M} X F_{\omega} \omega^{m}
$$

It is known that $f$ is independent of the choice of $\omega \in \Omega$. This implies that $f$ is invariant under automorphisms of $M$ and is a Lie algebra character. Obviously if $M$ admits a Kähler metric of constant scalar curvature then we have $F_{\omega}=0$ and $f$ vanishes.

Let $g$ be a Hodge metric, $\Omega$ its Kähler class, and $L$ a holomorphic line bundle with $c_{1}(L)=\Omega$. We assume that the action of an automorphism $\sigma$ lifts to $L$. Consider the character defined by

$$
\begin{aligned}
\hat{f}= & \frac{1}{2^{m+1}(m+1) !} \sum_{j=0}^{m}(-1)^{j}\left(\begin{array}{c}
m \\
j
\end{array}\right) \hat{f}_{K_{M}^{-1} \otimes L^{m-2 j}} \\
& -\frac{1}{2^{m+1}(m+1) !} \sum_{j=0}^{m}(-1)^{j}\left(\begin{array}{c}
m \\
j
\end{array}\right) \hat{f}_{K_{M} \otimes L^{m-2 j}} \\
& -\frac{\mu m}{2^{m+1}(m+1) !(m+1)} \sum_{j=0}^{m+1}(-1)^{j}\left(\begin{array}{c}
m+1 \\
j
\end{array}\right) \hat{f}_{L^{m+1-2 j}} .
\end{aligned}
$$

The following theorem has been proved by the second author [16].

Theorem 4.1. Let $X \in \mathfrak{h}(M)$. Then we have

$$
\mathfrak{R} f(X)=\left.\frac{d}{d t}\right|_{t=0} \hat{f}(\exp (t \Re X)) .
$$

where $\mathfrak{R} f(X)$ denotes the real part of $f(X)$.

The proof of this theorem will follow from later computations. Combining Remark 3.3 and Theorem 4.1 we obtain the following.

COROLLARY 4.2. The Lie algebra character $\mathfrak{R} f$ can be lifted to a group character $\hat{f}$ which can be written explicitly using Hermitian metrics of $L$ and $K_{M}$. Moreover $\hat{f}$ is independent of the choice of these metrics in each of the terms of $\hat{f}_{K_{M}^{-1} \otimes L^{m-2 j}}, \hat{f}_{K_{M} \otimes L^{m-2 j}}$ and $\hat{f}_{L^{m+1-2 j}}$.

In the rest of this paper we show that a suitable choice of Hermitian metrics of $L$ and $K_{M}^{-1}$ yields the K-energy, and when $L=K_{M}^{-1}$ a different choice yields Ding's functional.

Let $\omega$ be a Hodge metric of $M$ and $h$ an Hermitian metric of $L$. As before $\rho_{\omega}$ and $\rho_{h}$ respectively denotes the Ricci forms of $\omega$ and $h$. By the assumption we have $\left[\rho_{h}\right]=c_{1}(L)=[\omega]$. From Theorem 3.2 we have 
(5)

$$
\begin{aligned}
2 \pi \hat{f}(\sigma)= & \frac{1}{2^{m+1}(m+1) !} \sum_{j=0}^{m}(-1)^{j}\left(\begin{array}{c}
m \\
j
\end{array}\right) \int_{M}\left(\log \frac{\sigma^{*} \omega^{m}}{\omega^{m}}+(m-2 j) \log \frac{\sigma^{*} h}{h}\right) \\
& \sum_{k=0}^{m} \sigma^{*}\left(\rho_{\omega}+(m-2 j) \rho_{h}\right)^{k} \wedge\left(\rho_{\omega}+(m-2 j) \rho_{h}\right)^{m-k} \\
& -\frac{1}{2^{m+1}(m+1) !} \sum_{j=0}^{m}(-1)^{j}\left(\begin{array}{c}
m \\
j
\end{array}\right) \int_{M}\left(-\log \frac{\sigma^{*} \omega^{m}}{\omega^{m}}+(m-2 j) \log \frac{\sigma^{*} h}{h}\right) \\
& \sum_{k=0}^{m} \sigma^{*}\left(-\rho_{\omega}+(m-2 j) \rho_{h}\right)^{k} \wedge\left(-\rho_{\omega}+(m-2 j) \rho_{h}\right)^{m-k} \\
& -\frac{\mu m}{2^{m+1}(m+1) !(m+1)} \sum_{j=0}^{m+1}(-1)^{j}\left(\begin{array}{c}
m+1 \\
j
\end{array}\right)(m+1-2 j)^{m+1} \\
& \times \int_{M} \log \frac{\sigma^{*} h}{h} \sum_{k=0}^{m} \sigma^{*} \rho_{h}^{k} \wedge \rho_{h}^{m-k} .
\end{aligned}
$$

Using

$$
\begin{gathered}
\sum_{j=0}^{\ell}(-1)^{j}\left(\begin{array}{l}
\ell \\
j
\end{array}\right)(\ell-2 j)^{k}=0 \text { for } k \neq \ell, \\
\sum_{j=0}^{\ell}(-1)^{j}\left(\begin{array}{l}
\ell \\
j
\end{array}\right)(\ell-2 j)^{\ell}=2^{\ell} \ell !
\end{gathered}
$$

c.f. [19], and putting

$$
\gamma=\frac{\sigma^{*} \omega^{m}}{\omega^{m}}, \quad \tilde{\gamma}=\frac{\sigma^{*} h}{h}
$$

we obtain

(6) $\quad 2 \pi(m+1) \hat{f}(\sigma)=\frac{1}{2} \int_{M} \log \gamma \sum_{k=0}^{m} \sigma^{*} \rho_{h}^{k} \wedge \rho_{h}^{m-k}$

$$
\begin{aligned}
& +\log \tilde{\gamma} \sum_{k=0}^{m} k \sigma^{*} \rho_{\omega} \wedge \sigma^{*} \rho_{h}^{k-1} \wedge \rho_{h}^{m-k} \\
& +\log \tilde{\gamma} \sum_{k=0}^{m} \sigma^{*} \rho_{h}^{k} \wedge(m-k) \rho_{\omega} \wedge \rho_{h}^{m-k-1} \\
& -\frac{1}{2} \int_{M}(-\log \gamma) \sum_{k=0}^{m} \sigma^{*} \rho_{h}^{k} \wedge \rho_{h}^{m-k}
\end{aligned}
$$




$$
\begin{aligned}
& +\log \tilde{\gamma} \sum_{k=0}^{m} k\left(-\sigma^{*} \rho_{\omega}\right) \wedge \sigma^{*} \rho_{h}^{k-1} \wedge \rho_{h}^{m-k} \\
& +\log \tilde{\gamma} \sum_{k=0}^{m} \sigma^{*} \rho_{h}^{k} \wedge(m-k)\left(-\rho_{\omega}\right) \wedge \rho_{h}^{m-k-1} \\
& +\mu m \int_{M} \log \tilde{\gamma} \sum_{k=0}^{m} \sigma^{*} \rho_{h}^{k} \wedge \rho_{h}^{m-k} \\
& =\int_{M} \log \gamma \sum_{k=0}^{m} \sigma^{*} \rho_{h}^{k} \wedge \rho_{h}^{m-k}+\log \tilde{\gamma} \sum_{k=0}^{m} k \sigma^{*} \rho_{\omega} \wedge \sigma^{*} \rho_{h}^{k-1} \wedge \rho_{h}^{m-k} \\
& +\log \tilde{\gamma} \sum_{k=0}^{m} \sigma^{*} \rho_{h}^{k} \wedge(m-k) \rho_{\omega} \wedge \rho_{h}^{m-k-1} \\
& \quad-\mu m \int_{M} \log \tilde{\gamma} \sum_{k=0}^{m} \sigma^{*} \rho_{h}^{k} \wedge \rho_{h}^{m-k} .
\end{aligned}
$$

Suppose now that $\rho_{h}=\omega$. We put

$$
\varphi:=-\log \tilde{\gamma}=-\log \frac{\sigma^{*} h}{h} .
$$

Then we have

$$
\frac{i}{2 \pi} \partial \bar{\partial} \varphi=-\frac{i}{2 \pi} \partial \bar{\partial} \log \frac{\sigma^{*} h}{h}=\sigma^{*} \rho_{h}-\rho_{h}=\sigma^{*} \omega-\omega .
$$

So if we put $\omega_{\varphi}:=\sigma^{*} \omega$, then

$$
\omega_{\varphi}=\omega+\frac{i}{2 \pi} \partial \bar{\partial} \varphi \quad \text { and } \quad \sigma^{*} \rho_{\omega}=\rho_{\omega_{\varphi}} .
$$

Define a functional $\mathscr{M}\left(\omega, \omega_{\varphi}\right)$ by

$$
\mathscr{M}\left(\omega, \omega_{\varphi}\right)=\frac{2 \pi}{V} \hat{f}(\sigma) .
$$

THEOREM 4.3. $\mathscr{M}\left(\omega, \omega_{\varphi}\right)$ coincides with the K-energy.

Proof. From (6) we have

$$
\begin{aligned}
(m+1) V \mathscr{M}\left(\omega, \omega_{\varphi}\right)= & \int_{M} \log \frac{\omega_{\varphi}^{m}}{\omega^{m}} \sum_{k=0}^{m} \omega_{\varphi}^{k} \wedge \omega^{m-k}-\int_{M} \varphi \sum_{k=0}^{m} k \rho_{\omega_{\varphi}} \wedge \omega_{\varphi}^{k-1} \wedge \omega^{m-k} \\
& -\int_{M} \varphi \sum_{k=1}^{m+1} \omega_{\varphi}^{k-1} \wedge(m+1-k) \rho_{\omega} \wedge \omega^{m-k}
\end{aligned}
$$




$$
\begin{aligned}
& +\mu m \int_{M} \varphi \sum_{k=0}^{m} \omega_{\varphi}^{k} \wedge \omega^{m-k} \\
& =\int_{M} \log \frac{\omega_{\varphi}^{m}}{\omega^{m}} \sum_{k=0}^{m} \omega_{\varphi}^{k} \wedge \omega^{m-k} \\
& -(m+1) \int_{M} \varphi \sum_{k=1}^{m} \omega_{\varphi}^{k-1} \wedge \rho_{\omega} \wedge \omega^{m-k} \\
& \quad-\int_{M} \varphi \sum_{k=0}^{m} k\left(\rho_{\omega_{\varphi}}-\rho_{\omega}\right) \wedge \omega_{\varphi}^{k-1} \wedge \omega^{m-k} \\
& +\mu m \int_{M} \varphi \sum_{k=0}^{m} \omega_{\varphi}^{k} \wedge \omega^{m-k} .
\end{aligned}
$$

The third term of the right hand side is equal to

$$
\begin{aligned}
\int_{M} \log & \frac{\omega_{\varphi}^{m}}{\omega^{m}} \sum_{k=0}^{m} k\left(\omega_{\varphi}-\omega\right) \wedge \omega_{\varphi}^{k-1} \wedge \omega^{m-k} \\
= & \int_{M} \log \frac{\omega_{\varphi}^{m}}{\omega^{m}}\left(\omega_{\varphi} \wedge \omega^{m-1}-\omega^{m}+2\left(\omega_{\varphi}^{2} \wedge \omega^{m-2}-\omega_{\varphi} \wedge \omega^{m-1}\right)\right. \\
& \left.+\cdots+m\left(\omega_{\varphi}^{m}-\omega_{\varphi}^{m-1} \wedge \omega\right)\right) \\
= & -\int_{M} \log \frac{\omega_{\varphi}^{m}}{\omega^{m}}\left(\omega^{m}+\omega_{\varphi} \wedge \omega^{m-1}+\cdots+\omega_{\varphi}^{m}\right)+(m+1) \int_{M} \log \frac{\omega_{\varphi}^{m}}{\omega^{m}} \omega_{\varphi}^{m}
\end{aligned}
$$

Thus we obtain

$$
\begin{aligned}
V \mathscr{M}\left(\omega, \omega_{\varphi}\right)= & -\int_{M} \varphi \sum_{k=0}^{m-1} \omega_{\varphi}^{k} \wedge \rho_{\omega} \wedge \omega^{m-k-1} \\
& +\int_{M} \log \frac{\omega_{\varphi}^{m}}{\omega^{m}} \omega_{\varphi}^{m}+\frac{\mu m}{m+1} \int_{M} \varphi \sum_{k=0}^{m} \omega_{\varphi}^{k} \wedge \omega^{m-k} .
\end{aligned}
$$

This last expression is equal to the K-energy (c.f. [4], §5).

Proof of Theorem 4.1. Let $X \in \mathfrak{h}(M)$, and define $\varphi_{t}$ by

$$
(\exp (t \Re X))^{*} \omega-\omega=\frac{i}{2 \pi} \partial \bar{\partial} \varphi
$$

where $\mathfrak{R} X$ denotes the real part of $X$. Then 


$$
\begin{aligned}
\left.2 \pi \frac{d}{d t}\right|_{t=0} \hat{f}(\exp (t \Re X)) & =\left.\frac{d}{d t}\right|_{t=0} V \mathscr{M}\left(\omega, \omega_{\varphi}\right) \\
& =-\left.\int_{M} \dot{\varphi}\right|_{t=0}\left(s_{\omega}-\mu m\right) \omega^{m} \\
& =-\int_{M} \Delta\left(\left.\dot{\varphi}\right|_{t=0}\right) F_{\omega} \omega^{m} .
\end{aligned}
$$

Since $X$ is holomorphic, $\bar{\partial} i(X) \omega=0$. Hence the harmonic integration theory shows

$$
i(X) \omega=i \bar{\partial} u+i \alpha
$$

where $\alpha=\alpha_{j} d \bar{z}^{j}$ is a harmonic $(0,1)$-form. Therefore we have

$$
L_{1 / 2(X+\bar{X})} \omega=i \partial \bar{\partial}(\Re u) .
$$

It follows that

$$
\left.\dot{\varphi}\right|_{t=0}=\mathfrak{R} u
$$

modulo constant. Using $\bar{\partial}^{*} \alpha=0$, we have

$$
\begin{aligned}
-\int_{M} \Delta\left(\left.\dot{\varphi}\right|_{t=0}\right) F_{\omega} \omega^{m} & =-\int_{M} \Delta(\mathfrak{R}(u)) F_{\omega} \omega^{m} \\
& =-\int_{M} \mathfrak{R}(\operatorname{div}(X)) F_{\omega} \omega^{m} \\
& =2 \pi \mathfrak{R}(f(X)) .
\end{aligned}
$$

This completes the proof of Theorem 4.1.

Suppose that $c_{1}(M)>0$ and that $\rho_{\omega}=\omega+(i / 2 \pi) \partial \bar{\partial} F_{\omega}$. Then we have

$$
\begin{aligned}
\mathscr{M}\left(\omega, \omega_{\varphi}\right)= & -\frac{1}{V} \int_{M} \varphi \sum_{k=0}^{m-1} \omega_{\varphi}^{k} \wedge\left(\omega+\frac{i}{2 \pi} \partial \bar{\partial} F_{\omega}\right) \wedge \omega^{m-k-1}+\frac{1}{V} \int_{M} \log \frac{\omega_{\varphi}^{m}}{\omega^{m}} \omega_{\varphi}^{m} \\
& +\frac{m}{(m+1) V} \int_{M} \varphi \sum_{k=0}^{m} \omega_{\varphi}^{k} \wedge \omega^{m-k} \\
= & -\frac{1}{(m+1) V} \int_{M} \varphi \sum_{k=0}^{m} \omega_{\varphi}^{k} \wedge \omega^{m-k}+\frac{1}{V} \int_{M} \log \frac{\omega_{\varphi}^{m}}{\omega^{m}} \omega_{\varphi}^{m} \\
& -\frac{1}{V} \int_{M} F_{\omega}\left(\omega_{\varphi}^{m}-\omega^{m}\right)+\frac{1}{V} \int_{M} \varphi \omega_{\varphi}^{m} .
\end{aligned}
$$

This last expression is the K-energy for Fano manifolds used in [20].

Suppose again that $c_{1}(M)>0$ and that $L=K_{M}^{-1}$. We choose $h=\omega^{m}$. Then from (6) we have 


$$
2 \pi \hat{f}(\sigma)=\int_{M} \log \frac{\omega_{\varphi}^{m}}{\omega^{m}} \sum_{k=0}^{m} \rho_{\omega_{\varphi}}^{k} \wedge \rho_{\omega}^{m-k},
$$

where we have put $\omega_{\varphi}:=\sigma^{*} \omega$. We assume $\eta:=\rho_{\omega}>0, \eta^{\prime}:=\rho_{\omega_{\varphi}}>0$, and put $\eta^{\prime}=\eta+(i / 2 \pi) \partial \bar{\partial} \psi$. Then of course $\psi=-\log \omega_{\varphi}^{m} / \omega^{m}$, and thus

$$
2 \pi \hat{f}(\sigma)=-\int_{M} \psi \sum_{k=0}^{m} \eta^{\prime k} \wedge \eta^{m-k}
$$

Here $\psi$ is normalized by

$$
\int_{M} e^{-\psi} \omega^{m}=V
$$

We wish to rewrite the right hand side of (8) in the form invariant under the change of $\psi$ into $\psi+$ constant. If we define $F_{\eta}$ by

$$
\rho_{\eta}=\eta+\frac{i}{2 \pi} \partial \bar{\partial} F_{\eta}, \quad \int_{M} e^{F_{\eta}} \eta^{m}=V
$$

then

$$
F_{\eta}=\log \frac{\omega^{m}}{\eta^{m}}
$$

and

$$
\frac{1}{V} \int_{M} e^{-\psi+F_{\eta}} \eta^{m}=1
$$

Hence

$$
\frac{2 \pi}{V} \hat{f}(\sigma)=-\frac{1}{(m+1) V} \int_{M} \psi \sum_{k=0}^{m} \eta^{\prime k} \wedge \eta^{m-k}-\log \left(\frac{1}{V} \int_{M} e^{-\psi+F_{\eta}} \eta^{m}\right) .
$$

We define

$$
\mathscr{F}\left(\omega, \omega_{\varphi}\right)=-\frac{1}{(m+1) V} \int_{M} \varphi \sum_{k=0}^{m} \omega_{\varphi}^{k} \wedge \omega^{m-k}-\log \left(\frac{1}{V} \int_{M} e^{-\varphi+F_{\omega}} \omega^{m}\right),
$$

where $F_{\omega}$ is normalized by $1 / V \int_{M} e^{F_{\omega}} \omega^{m}=1$.

THEOREM 4.4. $\mathscr{F}\left(\omega, \omega_{\varphi}\right)$ coincides with Ding's functional.

Proof. Ding's functional ([8]), which we denote by $\mathscr{D}\left(\omega, \omega_{\varphi}\right)$, is defined by

$$
\mathscr{D}\left(\omega, \omega_{\varphi}\right)=J_{\omega}(\varphi)-\frac{1}{V} \int_{M} \varphi \omega^{m}-\log \left(\frac{1}{V} \int_{M} e^{F_{\omega}-\varphi} \omega^{m}\right),
$$

where $J_{\omega}(\varphi)$ is defined by 


$$
\begin{gathered}
I_{\omega}(\varphi)=\frac{1}{V} \int_{M} \varphi\left(\omega^{m}-\omega_{\varphi}^{m}\right), \\
J_{\omega}(\varphi)=\int_{0}^{1} \frac{I_{\omega}(s \varphi)}{s} d s .
\end{gathered}
$$

But one computes

$$
\begin{aligned}
J_{\omega}(\varphi) & =\frac{1}{V} \int_{0}^{1} \int_{M} \varphi\left(\omega^{m}-\omega_{s \varphi}^{m}\right) d s \\
& =\frac{1}{V} \int_{M} \varphi \omega^{m}-\frac{1}{V} \int_{0}^{1} \int_{M} \varphi\left(\omega+\frac{i}{2 \pi} \partial \bar{\partial} s \varphi\right)^{m} d s \\
& =\frac{1}{V} \int_{M} \varphi \omega^{m}-\frac{1}{(m+1) V} \int_{M} \varphi \sum_{k=0}^{m} \omega_{\varphi}^{m-k} \wedge \omega^{k} .
\end{aligned}
$$

This completes the proof.

\section{REFERENCES}

[1] T. Aubin, Equations du type de Monge-Ampère sur les variétés kählériennes compactes, C. R. Acad. Sci. Paris Sér. I Math., 283 (1976), 119-121.

[2] T. Aubin, Réduction du cas positif de l'équations de Monge-Ampère sur les variétés kählériennes compactes à la demonstration d'une inégalité, J. Funct. Anal., 57 (1984), 143-153.

[3] S. BANDO, An obstruction for Chern class forms to be harmonic, preprint, 1983.

[4] S. BANDO, The existence problem of Einstein-Kähler metrics: The case of positive scalar curvature, Sugaku, 50 (1998), 358-367, in Japanese; English translation, to appear from AMS.

[5] S. BANdo AND T. Mabuchi, Uniqueness of Einstein Kähler metrics modulo connected group actions, Algebraic Geometry, Sendai 1985, Adv. Stud. Pure Math. 10, Kinokuniya, Tokyo, 1987, 11-40.

[6] R. Вотт, Lectures on characteristic classes and foliations, Lectures on algebraic and differential topology, Lecture Notes in Math. 279, Springer, 1972, 1-94.

[7] E. CAlabI, Extremal Kähler metrics II, Differential Geometry and Complex Analysis, (I. Chavel and H. M. Farkas eds.), Springer, 1985, 95-114.

[ 8 ] W. Ding, Remarks on the existence problem of positive Kähler-Einstein metrics, Math. Ann., 282 (1988), 463-471.

[9] W. Ding AND G. Tian, Käler-Einstein metrics and the generalized Futaki invariant, Invent. Math., 110 (1992), 315-335.

[10] A. FutaKi, An obstruction to the existence of Einstein Kähler metrics, Invent. Math., 73 (1983), 437-443.

[11] A. Futaki, On compact Kähler manifold of constant scalar curvature, Proc. Japan Acad. Ser. A, 59 (1983), 401-402.

[12] A. FutAKI, Kähler-Einstein metrics and integral invariants, Lecture Notes in Math. 1314, Springer, 1988.

[13] T. MabuchI, K-energy maps integrating Futaki invariants, Tohoku Math. J., 38 (1987), 245257. 
[14] Y. Matsushima, Sur la structure du groupe d'homéomorphismes d'une certaine variété kaehlérienne, Nagoya Math. J., 11 (1957), 145-150.

[15] A. NADEL, Multiplier ideal sheaves and existence of Kähler-Einstein metrics of positive scalar curvature, Ann. of Math., 132 (1990), 549-596.

[16] Y. Nakagawa, The Bando-Calabi-Futaki character and its lifting to a group character, preprint.

[17] Y.-T. SIU, The existence of Kähler-Einstein metrics on manifolds with positive anticanonical line bundle and a suitable finite symmetry group, Ann. of Math., 127 (1988), 585-627.

[18] G. TIAN, Kähler-Einstein metrics on certain Kähler manifolds with $C_{1}(M)>0$, Invent. Math., 89 (1987), 225-246.

[19] G. Tian, Kähler-Einstein metrics on algebraic manifolds, Proc. C.I.M.E. conference on Transcendetal methods in algebraic geometry, Lecture Notes in Math. 1646, Springer, 1996, $143-185$.

[20] G. Tian, Kähler-Einstein metrics with positive scalar curvature, Invent. Math., 130 (1997), 137.

[21] S.-T. YAU, On Calabi's conjecture and some new results in algebraic geometry, Proc. Nat. Acad. Sci. USA, 74 (1977), 1798-1799.

Department of Mathematics

TOKyo InSTITUte of TeChNOLOGY

Meguro, TOKYO, 152-8551, JAPAN

Mathematical Institute

FACUTY OF SCIENCE

TOHOKU UNIVERSITY

SENDAI, 980-8578, JAPAN 